\title{
Performance of SDMA Systems Using Transmitter Preprocessing Based on Noisy Feedback of Vector-Quantized Channel Impulse Responses
}

\author{
Du Yang, Lie-Liang Yang and Lajos Hanzo \\ School of ECS, University of Southampton, SO17 1BJ, UK \\ Tel: +44-23-8059 3364; Fax: +44-23-8059 4508 \\ E-mail: dy05r,lly,lh@ecs.soton.ac.uk; http://www-mobile.ecs.soton.ac.uk
}

\begin{abstract}
In this contribution we investigate the performance of Spatia Division Multiple Access (SDMA) multiple-input multiple-output (MIMO) systems using transmitter preprocessing, when the channel knowledge required for preprocessing is acquired by the receiver and conveyed to the transmitter via noise feedback channels that may also conflict fading. Specifically, in our system the MIMO channel impulse responses (CIRs) are vector quantized. Then, the CIR magnitudes and phases are conveyed to the transmitter via a feedback channel, which is noise contaminated and may also experience Rayleigh fading. At the transmitter, the CIRs used for transmit preprocessing are recovered using a soft estimator, which is optimum in the minimum mean-square error (MMSE) sense, and is implemented based on the so-called Hadamard soft-decoding principles. Our study and simulation results demonstrate that vector quantization combined with soft-decoding constitutes an efficient technique of feeding back the CIRs from the receiver to the transmitter. However, it is also known that the performance of the zero-forcing (ZF) or MMSE transmit preprocessing schemes is highly sensitive to the effect of quantization errors as well as to the feedback channel induced errors.
\end{abstract}

\section{INTRODUCTION}

In recent years transmitter preprocessing techniques have received wide research attention [1]-[4], since the DownLink (DL) multiuser interference (MUI) may be mitigated by carrying out the required signal processing at the base-station (BS), where the associated complexity increase is less critical than at the mobile. Consequently, power-efficient and low-complexity mobile terminals (MTs) may be implemented. However, in order to carry out transmitter preprocessing at the BS for the sake of mitigating the MUI, the BS has to have the knowledge of all DL channels in advance, i.e. even before these channels are actually encountered by the transmitted DL signalling. In time-division duplex (TDD) systems, the knowledge of DL channels can be extracted from the UpLink (UL) channels, since in TDD mode the DL and UL TDD channels are reciprocal. However, in wireless systems using frequency-division duplexing (FDD), the employment of transmitter preprocessing becomes much more challenging in comparison to the TDD-type systems. This is because in FDD-type wireless systems the UL and DL channels are usually not reciprocal. Therefore, in FDD-type systems the DL CIRs would have to be estimated by the receiver and explicitly fed back from the MTs to the BS.

In contrast to [1]-[4], where ideal knowledge of the DL CIRs was assumed, in this contribution we consider the more realistic transmitter preprocessing scenario, when the BS has limited and possibly channel-infested knowledge about the downlink CIRs, which are conveyed through noise feedback channels experiencing fading. When transmitter preprocessing is carried out under the above-mentioned realistic conditions, it is important to design efficient CIR-signalling schemes and to investigate the impact of the recovered imperfect CIR knowledge on the achievable performance. In this contribution, realistic transmitter preprocessing using error-prone CIR signalling is investigated in the context of a SDMA system, which supports either single or multiple DL users. We assume that the CIRs between the BS transmit antennas and the MT's receive antennas are vector quantized (VQ) [5], where both the VQ codebook design and VQ code index assignment are considered. The bits representing the quantized CIRs are binary phase-shift keying (BPSK) modulated and conveyed to the BS transmitter through a fading channel impaired by additive Gaussian noise. At the BS's transmitter we assume that the CIRs used for transmit preprocessing are recovered using a soft-decoding scheme, in order to enhance the accuracy of CIR detection.

Note that one of our main objectives in this contribution is to quantify the number of bits required for representing the required channel information in the context of various feedback channel scenarios, so that the resultant bit error rate (BER) performance remains close to that achieved when the BS transmitter employs perfect knowledge of the DL CIRs.

\section{PRELiminaries}

\section{A. Transmitter Preprocessing}

Let us consider a MIMO system employing $N$ transmit antennas, which transmit a vector of $K$ symbols to the remote MT or MTs, where we assume that $N \geq K$. At the receiver side, there are a total of $K$ receive antennas, which may belong to one or several MTs. Consequently, when linear transmitter preprocessing is applied, it can be shown that the $K$-length vector $\boldsymbol{y}$ received by the $K$ antennas can be expressed as

$$
\boldsymbol{y}=\boldsymbol{H}^{T} \boldsymbol{P} \boldsymbol{x}+\boldsymbol{n}
$$

where $\boldsymbol{x}=\left[x_{1}, x_{2}, \ldots, x_{K}\right]^{T}$ contains the $K$ transmitted symbols. It is assumed that $E\left[\left|x_{k}\right|^{2}\right]=1$ and $x_{k}$ is an independently and identically distributed (iid) uniform random variable. In (1) $n$ is the $K$-length noise vector, hosting complex Gaussian random variables having a zero mean and a variance of $\sigma^{2} / 2=1 / 2$ SNR per dimension, where SNR represents the signal-to-noise ratio averaged over all the $N$ transmission channels. In (1) $\boldsymbol{P}$ is the $(N \times K)$ transmitter preprocessing matrix, which is given by

$$
\boldsymbol{P}=\left[\boldsymbol{p}_{1}, \boldsymbol{p}_{2}, \ldots, \boldsymbol{p}_{K}\right],
$$

where $\boldsymbol{p}_{k}$ represents a vector for preprocessing $x_{k}, k=1, \ldots, K$. Furthermore, in (1) the matrix $\boldsymbol{H}$ containing the CIRs between the transmit and receiver antennas can be expressed as

$$
\begin{aligned}
\boldsymbol{H} & =\left[\boldsymbol{h}_{1}, \boldsymbol{h}_{2}, \ldots, \boldsymbol{h}_{K}\right] \\
\boldsymbol{h}_{k} & =\left[h_{k 1}, h_{k 2}, \ldots, h_{k N}\right]^{T}, k=1,2, \ldots, K,
\end{aligned}
$$

where $\boldsymbol{h}_{k}$ is the CIR, which is often referred to as the spatial signature, corresponding to the $k$ th receive antenna, where $x_{k}$ is detected.

In this contribution three different-complexity preprocessing schemes are considered in our simulations, which are based on the principles of transmitter matched-filtering (TMF), transmitter zeroforcing (TZF) and transmitter minimum mean-square error (TMMSE) processing [1]-[4]. Specifically, in the context of TMF, the preprocessing matrix $P$ is given by [4]

$$
P=H^{*} \beta,
$$

where $\boldsymbol{\beta}=\operatorname{diag}\left\{\beta_{1}, \beta_{2}, \cdots, \beta_{K}\right\}$ is a matrix of normalization factors required for maintaining a constant transmitted power, which can be chosen to satisfy

$$
\operatorname{Tr}\left(\boldsymbol{P} \boldsymbol{P}^{H}\right)=K
$$


where $\operatorname{Tr}(\boldsymbol{X})$ represents the trace of $\boldsymbol{X}$. In the context of TZF-assisted preprocessing, the preprocessing matrix $\boldsymbol{P}$ is given by [2], [4]

$$
\boldsymbol{P}=\boldsymbol{H}^{*}\left(\boldsymbol{H}^{T} \boldsymbol{H}^{*}\right)^{-1} \boldsymbol{\beta} .
$$

Finally,the matrix $\boldsymbol{P}$ used for TMMSE-assisted preprocessing is given by [2]-[4]

$$
\boldsymbol{P}=\boldsymbol{H}^{*}\left(\boldsymbol{H}^{T} \boldsymbol{H}^{*}+\sigma^{2} \boldsymbol{I}_{K}\right)^{-1} \boldsymbol{\beta} .
$$

As seen by comparing (6) and (7), TZF carries out multiuser preprocessing without taking into account the effects of the background noise, while TMMSE jointly minimizes the effects of the MUI and of the background noise. Correspondingly, as shown in [3], [4], TZFassisted preprocessing suppresses the MUI at the cost of potentially amplifying the background noise.

\section{B. Vector Quantization}

Let us initially assume that the source $\left\{\boldsymbol{\alpha}_{n}\right\}$, which has to be quantized, namely the MIMO CIR vector, can be modeled by a zeromean, stationary and ergodic vector process. This MIMO CIR vector is encoded using vector quantization [5], which is described as follows. The VQ source encoder carries out the mapping $\mathcal{E}: \mathcal{R}^{V} \rightarrow \mathcal{I}_{U}$, which maps the $V$-dimensional unquantized, i.e. continuous valued source vector $\boldsymbol{\alpha}_{n} \in \mathcal{R}^{V}$ into a finite precision representation $I_{n} \in \mathcal{I}_{U}$, where we have $I_{n}=\mathcal{E}\left(\boldsymbol{\alpha}_{n}\right)$, while $\mathcal{I}_{U}=\{0,1, \ldots, U-1\}$ represents the $U$ number of VQ centroids. The encoder's mapping function $\mathcal{E}$ is defined by a VQ partition $\left\{\mathcal{R}_{i}\right\}_{i=0}^{U-1}$ of the $V$-dimensional MIMO CIR hyperspace $\mathcal{R}^{V}$, so that we have $\boldsymbol{\alpha}_{n} \in \mathcal{R}_{i} \Rightarrow I_{n}=i$. Hence, the unquantized MIMO CIR set $\mathcal{R}_{i}$ is quantized into the $i$ th VQ region or cell. Let us define the VQ-encoded centroids, $\left\{\boldsymbol{c}_{i}\right\}_{i=0}^{U-1}$ as $\boldsymbol{c}_{i} \triangleq E\left[\boldsymbol{\alpha}_{n} \mid I_{n}=i\right]=E\left[\boldsymbol{\alpha}_{n} \mid \boldsymbol{\alpha}_{n} \in \mathcal{R}_{i}\right]$. The VQ-centroid set $\left\{\boldsymbol{c}_{i}\right\}_{i=0}^{U-1}$ is stored in the VQ-encoded codebook, which has a size of $U$ entries, while $i \in \mathcal{I}_{U}$ is referred to as the index of the $i$ th codeword.

Let $b_{l}(i) \in\{-1,+1\}, l=0,1, \ldots, V-1$ be the bits in the binary representation of the integer VQ codebook index $i \in \mathcal{I}_{U}$. When $b_{l}(i)$ is transmitted via a feedback channel from a MT to the BS, the channel observation for $b_{l}(i)$ at the BS's receiver can be expressed as

$$
y_{l}^{(f)}(i)=h_{l}^{(f)} b_{l}(i)+n_{l}^{(f)}, l=0,1, \ldots, V-1,
$$

where the superscript $f$ indicates the feedback channel, $h_{l}^{(f)}$ represents the Rayleigh faded channel gain experienced by the $l$ th bit, while $n_{l}^{(f)}$ denotes the corresponding noise sample, which is assumed to be a Gaussian distributed random variable with zero-mean and a variance of $1 / 2 \mathrm{SNR}^{(f)}$ per dimension.

Note that, in this contribution we investigate the performance of transmit preprocessing schemes using finite-precision channel knowledge at the transmitter, when assuming that the elements of $\boldsymbol{H}$ in (3) are either independent or correlated in the spatial-domain. For the scenario when the elements of $\boldsymbol{H}$ are independent and complex Gaussian distributed, the MIMO channel's CIR matrix $\boldsymbol{H}$ can be readily generated with the aid of the complex Gaussian distribution. By contrast, when the elements of the MIMO CIR matrix $\boldsymbol{H}$ are correlated, in our simulations the correlated MIMO CIR matrix $\boldsymbol{H}$ is generated using the approach proposed in [6]. This approach creates the spatially correlated MIMO channels using the virtual channel representation (VCR) associated with the 2-dimensional Discrete Fourier Transform (DFT).

\section{MIMO CIR QUANTIZATION AND CODEBooK DESIGN}

During our initial study we found that when the CIRs are vector quantized, the phases and amplitudes should be quantized separately using two vectors. The reason for the above observation is that the phase and amplitude are distributed significantly differently. More explicitly, the former is uniformly distributed, while the latter is assumed to be Rayleigh distributed in this contribution. Furthermore, in our initial studies, the BER performance of SDMA systems using transmitter preprocessing was found to be more sensitive to phase errors than to amplitude errors, hence the phase potentially requires a more accurate VQ.

Our codebooks are generated using the generalized Lloyd algorithm [5], which is described as follows:

Step 1 : Generate a sufficiently large number of $M$ empirical data samples $\boldsymbol{X}=\left[\boldsymbol{\alpha}_{1}, \boldsymbol{\alpha}_{2}, \ldots, \boldsymbol{\alpha}_{M}\right]$, where $\boldsymbol{\alpha}_{i}$ is $V$-dimensional and represents either the amplitudes or phases of the MIMO CIR matrix $H$ to be quantized;

Step 2: Set $m=1$ and randomly choose $U$ columns in the empirical data $\boldsymbol{X}$ as the initial codebook $\boldsymbol{C}_{m}$, which can be expressed as

$$
\boldsymbol{C}_{m}=\left[\boldsymbol{c}_{0}^{(m)}, \boldsymbol{c}_{1}^{(m)}, \ldots, \boldsymbol{c}_{U-1}^{(m)}\right]
$$

Step 3 : Use Lloyd procedure for updating the codebook $\boldsymbol{C}_{m}$ to generate $\boldsymbol{C}_{m+1}$ as follows:

(a) Associate each column in $\boldsymbol{X}$ with a codeword in $\boldsymbol{C}_{m}$. Specifically, a vector $\boldsymbol{\alpha}_{t}$ in $\boldsymbol{X}$ is associated with $\boldsymbol{c}_{i}^{(m)}$, if it satisfies

$$
d\left(\boldsymbol{\alpha}_{t}, \boldsymbol{c}_{i}^{(m)}\right)<d\left(\boldsymbol{\alpha}_{t}, \boldsymbol{c}_{j}^{(m)}\right) ; \text { for all } j \neq i,
$$

where $d(\cdot, \cdot)$ represents the Euclidean distance of the arguments. Provided that $d\left(\boldsymbol{\alpha}_{t}, \boldsymbol{c}_{i}^{(m)}\right)=d\left(\boldsymbol{\alpha}_{t}, \boldsymbol{c}_{j}^{(m)}\right)$, then $\boldsymbol{\alpha}_{t}$ is associated with $\boldsymbol{c}_{i}^{(m)}$ if $i \leq j$, otherwise, it is associated with $\boldsymbol{c}_{j}^{(m)}$ if $i>j$;

(b) All the unquantized CIR vectors $\left\{\boldsymbol{\alpha}_{i}\right\}$ that will be represented by the same VQ codeword, say $\boldsymbol{c}_{i}^{(m)}$, form a VQ cell referred to as $\mathcal{R}_{i}, i=0,1, \ldots, U-1$;

(c) Based on the updated cells $\left\{\mathcal{R}_{i}\right\}$, recompute the VQ centroids representing the quantized CIRs in the context of all the VQ cells, and correspondingly update the codebook to $\boldsymbol{C}_{m+1}=\left[\boldsymbol{c}_{0}^{(m+1)}, \boldsymbol{c}_{1}^{(m+1)}, \ldots, \boldsymbol{c}_{U-1}^{(m+1)}\right]$;

Step 4 : Based on the updated codebook $\boldsymbol{C}_{m+1}$, compute the VQ scheme's overall distortion measured in terms of its meansquare error (MSE), which is expressed as

$$
D_{m+1}=\frac{1}{M} \sum_{i=0}^{U-1} \sum_{\boldsymbol{\alpha}_{j} \in \mathcal{R}_{i}}\left|\boldsymbol{\alpha}_{j}-\boldsymbol{c}_{i}^{(m+1)}\right|^{2}
$$

Step 5 : If the relative distortion reduction $\left|D_{m+1}-D_{m}\right|$ achieved during the $m$ th VQ training step is sufficiently small, curtail the iterations and use $\boldsymbol{C}_{m+1}$ as the codebook $\boldsymbol{C}=$ $\left[\boldsymbol{c}_{0}, \boldsymbol{c}_{1}, \ldots, \boldsymbol{c}_{U-1}\right]$. Otherwise, set $m+1 \rightarrow m$ and repeat the process from Step 3.

After obtaining the codebook $\boldsymbol{C}=\left[\boldsymbol{c}_{0}, \boldsymbol{c}_{1}, \ldots, \boldsymbol{c}_{U-1}\right]$, the indices of the VQ codewords can be re-ordered, so that the quantized CIR VQ codewords exhibiting similarity are represented by indices having similar binary representations. When the codeword indices are arranged in this way, the effect of transmission errors caused by noise and fading imposed by the transmission channels can be minimized.

Figs. 1 and 2 show the codeword distributions for the amplitudes and phases of two spatially correlated MIMO channels having a correlation coefficient of $\rho \approx 0.8$, when vector quantization based on the abovementioned algorithm is employed. Both the amplitudes and phases were quantized into 1024 legitimate codewords. Correlated Rayleigh fading channels having a correlation coefficient of $\rho \approx 0.8$ were assumed. It can be observed that the codewords representing both the amplitudes and phases tend to be indeed closely correlated.

\section{Soft-Decoding Assisted MIMO CIR ReCOVERY}

We assume that the VQ index, say $I_{n}=i$ representing $\boldsymbol{\alpha}_{n}$, and corresponding to a specific CIR tap at a certain time instant is fed back to the BS's transmitter via a feedback channel. When BPSK 


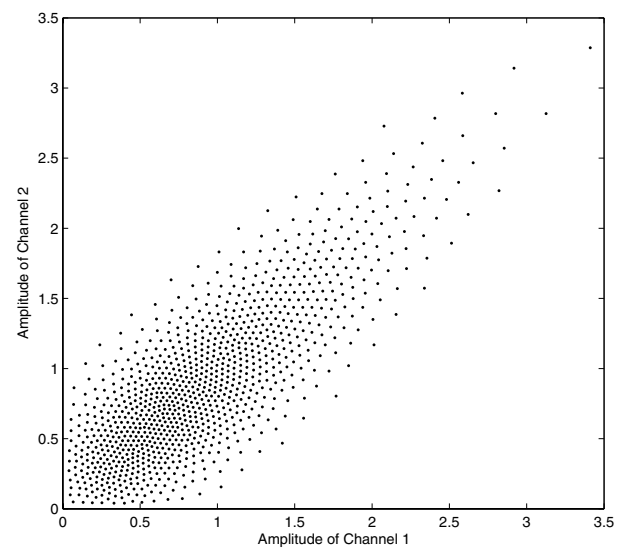

Fig. 1. Illustration of the 1024 codewords for the amplitudes of two spatial correlated MIMO channels having a correlation coefficient of $\rho \approx 0$. vector quantization is employed.

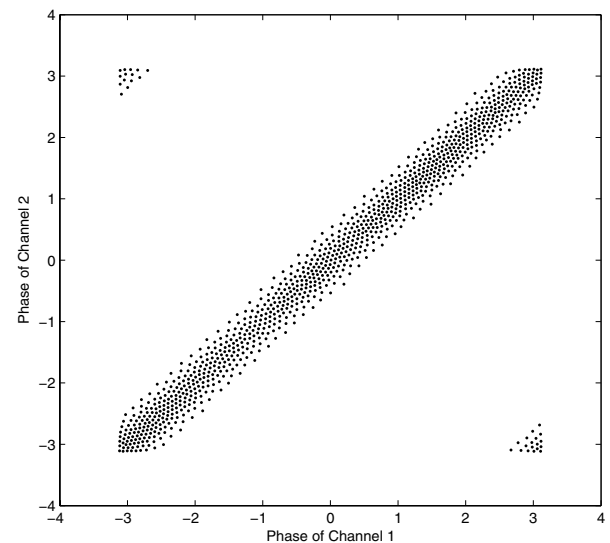

Fig. 2. Illustration of the 1024 codewords for the phases of two spatially correlated MIMO channels having a correlation coefficient of $\rho \approx 0.8$, when vector quantization is employed.

modulation is assumed, the observations for the VQ index $I_{n}=i$ at the BS's transmitter can be expressed as

$$
y_{l}^{(f)}(i)=h_{l}^{(f)} b_{l}(i)+n_{l}^{(f)}, l=0,1, \ldots, V-1,
$$

where $V$ is the number of bits required by the binary representation of $I_{n}=i$, which is expressed as $\boldsymbol{b}_{i}=\left\{b_{V-1}(i), \cdots, b_{0}(i)\right\}$, $h_{l}^{(f)}$ represents the fading gain, while $n_{l}^{(f)}$ is the Gaussian noise. Furthermore, provided that the phase associated with a specific fading gain $h_{l}^{(f)}$ is known, after removing the effects of the phase, (11) can be expressed as

$$
y_{l}^{(f)}(i)=\left|h_{l}^{(f)}\right| b_{l}(i)+n_{l}^{(f)}, l=0,1, \ldots, V-1 .
$$

The received CIRs of the MIMO channel are recovered with the aid of a soft source decoder, which is a non-linear MMSE estimator [7]. Its objective is to compute the estimate $\hat{\boldsymbol{\alpha}}_{n}$ of $\boldsymbol{\alpha}_{n}$, based on the observations of $y_{l}^{(f)}(i)$ for $l=0,1, \ldots, V-1$, where $\boldsymbol{\alpha}_{n}$ represents either a set of amplitudes or a set of phases. Let the vector

$$
\boldsymbol{y}^{(f)}=\left\{y_{0}^{(f)}(i), y_{1}^{(f)}(i), \ldots, y_{V-1}^{(f)}(i)\right\}
$$

host the $V$ observations corresponding to $\boldsymbol{\alpha}_{n}$. Then, $\boldsymbol{\alpha}_{n}$ is estimated by the MMSE estimator according to [7]

$$
\begin{aligned}
\hat{\boldsymbol{\alpha}}_{n} & =\sum_{j=0}^{U-1} E\left[\boldsymbol{\alpha}_{n} \mid I_{n}=j\right] P\left(I_{n}=j \mid \boldsymbol{y}^{(f)}\right) \\
& =\sum_{j=0}^{U-1} \boldsymbol{c}_{j} P\left(I_{n}=j \mid \boldsymbol{y}^{(f)}\right),
\end{aligned}
$$

where $\boldsymbol{c}_{j}=E\left[\boldsymbol{X}_{n} \mid I_{n}=j\right], j=0,1, \ldots, U-1$, is the codeword or centroid of the $j$ th VQ partition described in Section III. In (14) $P\left(I_{n}=j \mid \boldsymbol{y}^{(f)}\right)$ represents the a-posteriori probability of the VQ codebook index $I_{n}=j$ given the observation vector of $\boldsymbol{y}^{(f)}$, which can be expressed as

$$
P\left(I_{n}=j \mid \boldsymbol{y}^{(f)}\right)=\frac{P\left[I_{n}=j\right] p\left[\boldsymbol{y}^{(f)} \mid I_{n}=j\right]}{p\left(\boldsymbol{y}^{(f)}\right)},
$$

where $P\left[I_{n}=j\right]$ is the a-priori probability of the event $I_{n}=j$, which can be found according to the specific channel statistics encountered, while $p\left[\boldsymbol{y}^{(f)} \mid I_{n}=j\right]$ is the probability density function (PDF) of the event, when the natural binary representation of $I_{n}=j$ is transmitted over the feedback channel. Assuming that the consecutive samples of the MIMO feedback channel's CIRs encountered during the transmission of the VQ bits are independent of each other, we can express it as

$$
p\left[\boldsymbol{y}^{(f)} \mid I_{n}=j\right]=\prod_{l=0}^{V-1} p\left[y_{l}^{(f)}(i) \mid b=b_{l}(j)\right],
$$

where $p\left[y_{l}^{(f)}(i) \mid b_{l}(j)\right]$ is the probability density of observing $y_{l}^{(f)}(i)$, given that $b=b_{l}(j)$ was transmitted, which can be expressed as

$$
p_{y_{l}^{(f)}(i)}\left[y \mid b=b_{l}(j)\right]=\frac{1}{\sqrt{2 \pi} \sigma} \exp \left(-\frac{\left[y-\left|h_{l}^{(f)}\right| b_{l}(j)\right]^{2}}{2 \sigma^{2}}\right),
$$

where $\sigma^{2}$ is the variance of the Gaussian noise.

Finally, in (15) $p\left(\boldsymbol{y}^{(f)}\right)$ is the probability density of the observation $\boldsymbol{y}^{(f)}$, which can be expressed as

$$
p\left(\boldsymbol{y}^{(f)}\right)=\sum_{j=0}^{U-1} P\left[I_{n}=j\right] p\left[\boldsymbol{y}^{(f)} \mid I_{n}=j\right] .
$$

In our simulations the so-called Hadamard soft-decoding technique [8] is employed for implementing the above-mentioned MMSE estimation in procedure of [7] in terms of the individual bits representing the transmitted VQ index [8]. To be more specific, with the aid of the Hadamard soft-decoding technique of [7], the $i$ th VQ centroid can be expressed as

$$
c_{i}=T m_{i},
$$

where $\boldsymbol{m}_{i}$ is the $i$ th column of a $(U \times U)$ Sylvester-type Hadamard matrix $\boldsymbol{M}$ [8]. To elaborate a little further, let us express the natural binary representation of the integer $i$ as $\boldsymbol{b}_{i}=\left\{b_{V-1}(i), \cdots, b_{0}(i)\right\}$. Then, it can be shown that the $i$ th column $\boldsymbol{m}_{i}$ in $\boldsymbol{M}$ can be generated as [8]

$$
\boldsymbol{m}_{i}=\left[\begin{array}{c}
1 \\
b_{V-1}(i)
\end{array}\right] \otimes\left[\begin{array}{c}
1 \\
b_{V-2}(i)
\end{array}\right] \otimes \cdots \otimes\left[\begin{array}{c}
1 \\
b_{0}(i)
\end{array}\right] .
$$

In (19) $T$ is the Hadamard transform matrix which is fully determined by the encoder centroids and can be expressed as [8]

$$
T=\frac{1}{U} C M,
$$


where $C$ is the codebook obtained according to Section III.

Upon substituting (19) into (14), the MMSE estimate $\hat{\boldsymbol{\alpha}}_{n}$ of $\boldsymbol{\alpha}_{n}$ can be expressed as

$$
\hat{\boldsymbol{\alpha}}_{n}=\boldsymbol{T} \sum_{j=0}^{U-1} \boldsymbol{m}_{j} P\left(I_{n}=j \mid \boldsymbol{y}^{(f)}\right) .
$$

Following a number of further steps and by referring to Section III, $\hat{\boldsymbol{\alpha}}_{n}$ can be expressed as

$$
\hat{\boldsymbol{\alpha}}_{n}=\boldsymbol{T}\left[\frac{\boldsymbol{R}_{\boldsymbol{m}} \boldsymbol{q}\left(\boldsymbol{y}^{(f)}\right)}{\boldsymbol{r}_{\boldsymbol{m}}^{T} \boldsymbol{q}\left(\boldsymbol{y}^{(f)}\right)}\right],
$$

where we have

$$
\begin{aligned}
\boldsymbol{R}_{\boldsymbol{m}} & =\sum_{i=0}^{U-1} P\left(I_{n}=i\right) \times \boldsymbol{m}_{i} \boldsymbol{m}_{i}^{T} \\
\boldsymbol{r}_{\boldsymbol{m}} & =\sum_{i=0}^{U-1} P\left(I_{n}=i\right) \times \boldsymbol{m}_{i} .
\end{aligned}
$$

Furthermore, $\boldsymbol{q}\left(\boldsymbol{y}^{(f)}\right)$ in (23) can be expressed as

$$
\boldsymbol{q}\left(\boldsymbol{y}^{(f)}\right)=\left[\begin{array}{c}
1 \\
\hat{b}_{V-1}
\end{array}\right] \otimes\left[\begin{array}{c}
1 \\
\hat{b}_{V-2}
\end{array}\right] \otimes \cdots \otimes\left[\begin{array}{c}
1 \\
\hat{b}_{0}
\end{array}\right],
$$

where $\hat{b}_{k}, k=0,1, \ldots, V-1$ represents the a-posteriori estimation of $b_{k}$, which is given by

$$
\begin{aligned}
\hat{b}_{k}=E\left[b_{k} \mid y_{l}^{(f)}(i)\right]_{P\left(b_{k}= \pm 1\right)=0.5} \\
=P\left[b_{k}=+1 \mid y_{l}^{(f)}(i)\right]-P\left[b_{k}=-1 \mid y_{l}^{(f)}(i)\right] \\
=\frac{1}{2 p\left(y_{l}^{(f)}(i)\right)}\left(p_{y_{l}^{(f)}{ }_{(i)}}\left[y \mid b_{k}=+1\right]\right. \\
\left.-p_{y_{l}^{(f)}(i)}\left[y \mid b_{k}=-1\right]\right),
\end{aligned}
$$

while $p_{y_{l}^{(f)}(i)}\left[y \mid b_{k}=+1\right]$ and $p_{y_{l}^{(f)}{ }_{(i)}}\left[y \mid b_{k}=-1\right]$ can be expressed from (17). Furthermore $p\left(y_{l}^{(f)}(i)\right)$ is given by

$$
p\left(y_{l}^{(f)}(i)\right)=\frac{1}{2} \sum_{b= \pm 1} p_{y_{l}^{(f)}(i)}\left[y \mid b_{k}=b\right] .
$$

Let us now provide some performance results in the next section.

\section{Performance Results AND CONClusions}

Figs. 3 and 4 show the BER performance of the multiple-input single-output (MISO) system using four transmit antennas and a single receive antenna, when communicating over the four correlated Rayleigh fading channels having a correlation coefficient of $\rho \approx 0.8$. In this example four specific scenarios concerning the knowledge of the CIR used for transmit preprocessing were considered. 1) The first scenario assumes that the transmitter employs perfect CIR knowledge for preprocessing. 2) The second investigation assumes that finiteprecision, but instantaneous CIR knowledge is conveyed via an ideal feedback channel imposing no noise and no fading. 3) By contrast, the third and fourth cases assume that instantaneous, finite-precision CIR knowledge is fed back from the receiver to the transmitter via an imperfect feedback channel, which is either a Gaussian channel or an AWGN-contaminated Rayleigh fading channel. In our simulations characterized in Figs. 3 and 4 we assume that both the amplitudes and phases of the non-dispersive CIR taps are ideally estimated, both of which are quantized using $V=12$ bits corresponding to $U=4096$ VQ codewords. Furthermore, for the feedback channel the signals received by the BS's four receive antennas are coherently diversity

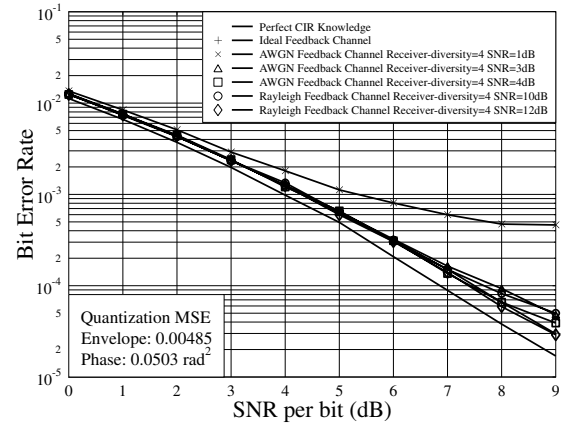

Fig. 3. BER versus SNR per bit performance of a MISO channel using four transmit antennas and a single receive antenna, when communicating over independent Rayleigh fading MIMO channels, where 12 bits are used for quantizing both the amplitudes and phases, respectively.

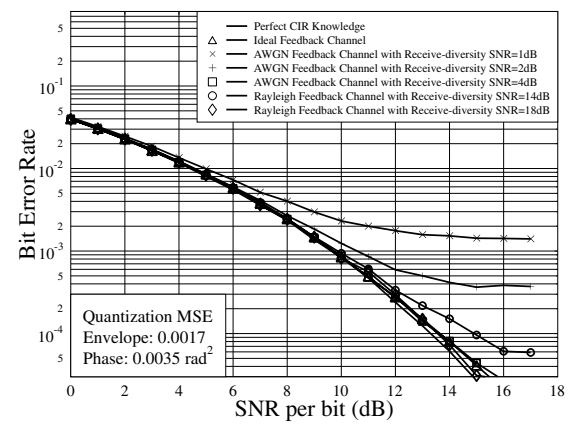

Fig. 4. BER versus SNR per bit performance of a MISO channel using four transmit antennas and a single receive antenna, when communicating over correlated Rayleigh fading MIMO channels associated with $\rho \approx 0.8$, where 12 bits are used for quantizing both the amplitudes and phases, respectively.

combined, so that the CIRs can be estimated more reliably. Observe from the results of Fig.3, that if the four channels corresponding to the four transmit antennas are independent, using $U=4096$ codewords for the quantization of both the amplitudes and phases is still insufficiently accurate. As shown in Fig.3, an approximately 0.5 $\mathrm{dB}$ of SNR loss is experienced at the BER of $10^{-4}$ by VQ scheme. By contrast, when the four transmission channels are correlated, as shown in Fig. 4, the BER performance achieved over a wide SNR range is close to that obtained by using perfect CIR knowledge for transmitter preprocessing. From the results of Figs. 3 and 4 we infer that a Gaussian feedback channel having a SNR of $4 \mathrm{~dB}$ is sufficiently reliable for conveying the CIR information. By contrast, for Rayleigh fading feedback channels, the SNR required for reliably conveying the channel information is about $12 \mathrm{~dB}$, when the four receivediversity channels are independently fading, as evidenced by Fig.3. When the four channels are correlated, the required SNR becomes $18 \mathrm{~dB}$, as shown in Fig. 4. The reason for this is that uncorrelated fading results in a higher diversity gain for the feedback channel than experienced in the correlated fading scenario. Furthermore, when comparing the results of Figs. 3 to those of Fig. 4, it can be observed that the MIMO system experiencing independent fading outperforms that experiencing correlated fading. Again, this is because a significant diversity gain loss is encountered due to the correlation among the MIMO transmission channels.

Figs. 5 and Figs. 6 show the BER performance of the MIMO system 


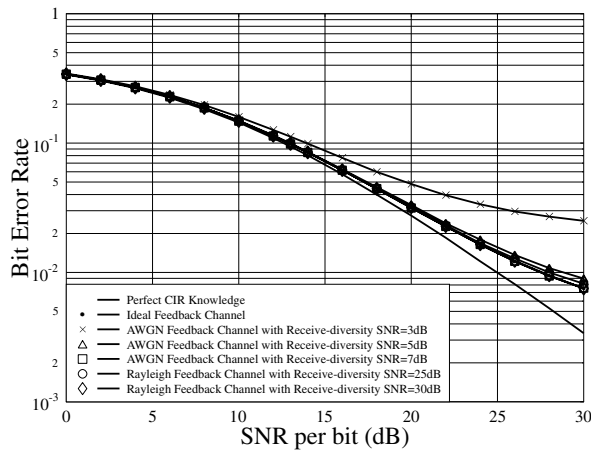

Fig. 5. TZF: BER versus SNR per bit performance of a MIMO channel using two transmit antennas, two receive antennas and supporting two users, when communicating over correlated Rayleigh fading channels associated with $\rho \approx$ 0.8 , where 10 bits are used for quantizing both the amplitudes and phases, respectively, of each user.



Fig. 6. TMMSE: BER versus SNR per bit performance of a MIMO channel using two transmit antennas, two receive antennas and supporting two users, when communicating over correlated Rayleigh fading channels associated with $\rho \approx 0.8$, where 10 bits are used for quantizing both the amplitudes and phases, respectively, of each user.

using two transmit antennas, two receive antennas and supporting two users, while employing the soft decoding aided recovery of the VQ indices over noisy channels for either the TZF (Fig. 5) or for the TMMSE (Fig. 6) based preprocessing schemes mentioned in section II, when communicating over correlated Rayleigh fading MIMO channels having a correlation coefficient of $\rho \approx 0.8$. The results of Figs. 5 and 6 demonstrate that as expected, VQ of the CIR results in a performance loss, when the downlink SNR is relatively high. When the downlink SNR is below $15 \mathrm{~dB}$, the performance loss imposed by the vector quantization of the CIR is hardly noticeable.

Finally, in Fig. 7 we compare the attainable BER performance, when using TMF, TZF and TMMSE-assisted transmitter preprocessing. Specifically, we assumed a MIMO system using $N_{t}=4$ transmit antennas, $N_{r}=4$ receive antennas and supporting $K=4$ users, when communicating over correlated Rayleigh fading channels having a correlation coefficient of $\rho \approx 0.8$. The results seen in Fig. 7 reflect how sensitive the BER performance achieved by the transmitter preprocessing schemes is to the non-ideal CIR knowledge. As the results seen in Fig. 7 demonstrate, the TMF scheme achieves the worst BER performance among the preprocessing schemes considered. However, it is nonetheless a robust preprocessing scheme, hence its performance

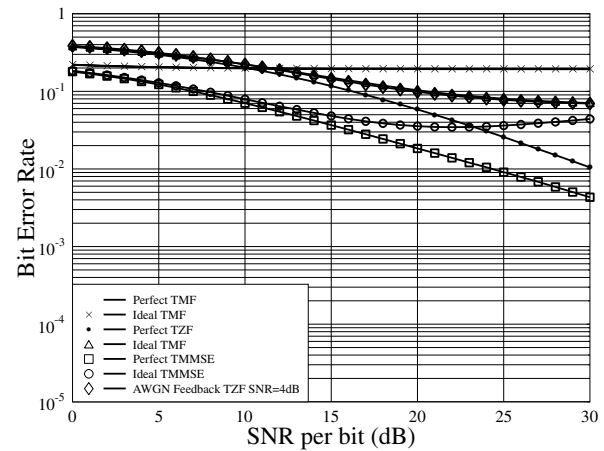

Fig. 7. Performance comparison of the TMF, TZF and soft decoding for vector quantization over noisy channels with memory TMMSE assisted preprocessing schemes for the MIMO system using 4 transmit antennas, 4 receive antennas and supporting 4 users, when communicating over correlated Rayleigh fading channels associated with $\rho \approx 0.8$, where 12 bits are used for quantizing both the amplitudes and phases of each user.

does not degrade significantly upon increasing the channel estimation error. By contrast, both the TZF and TMMSE assisted preprocessing schemes are highly sensitive to the accuracy of the CIR knowledge, especially, in the relatively high SNR region, where a significant BER performance degradation can be observed.

In conclusion, we have investigated the achievable performance of SDMA MIMO systems using various transmitter preprocessing schemes, when the CIR knowledge used for preprocessing is vector quantized and conveyed via noise feedback channels from the MT's receivers to the BS's transmitter. Our study demonstrated that vector quantization combined with soft-decoding is an efficient technique of signalling the CIR knowledge to the BS's transmitter. However, our study also shows that the performance of the TZF and TMMSE transmitter preprocessing schemes are highly sensitive to the non-ideal CIR knowledge.

\section{ACKNOWLEDGEMENT}

The author would like to acknowledge the financial assistance of the EPSRC of UK, and of the EU under the auspices of the Newcom and Phonix projects.

\section{REFERENCES}

[1] R. Esmailzadeh, E. Sourour, and M. Nakagawa, "Prerake diversity combining in time-division duplex CDMA mobile communications," IEEE Trans. on Veh. Tech., vol. 48, no. 3, pp. 795 - 801, May 1999.

[2] B. Vojcic and W. Jang, "Transmitter preprocessing in synchronous multiuser communications," IEEE Transaction on Communications, vol. 46, no. 10 , pp. 1346-1355, Oct. 1998.

[3] R. L.-U. Choi and R. D. Murch, "New transmit schemes and simplified receivers for MIMO wireless communication systems," IEEE Transaction on Wireless Communications, vol. 2, no. 6, pp. 1217-1230, Nov. 2003.

[4] M. Joham, W. Utschick, and J. A. Nossek, "Linear transmit processing in MIMO communications systems," IEEE Transaction on Signal Processing, vol. 53 , no. 8 , pp. $2700-2712$, Aug. 2005

[5] A. Gersho and R. M. Gray, Vector Quantization and Signal Compression. Boston/Dordrecht/London: Kluwer Academic Publishers, 1991

[6] H. Tong and S. A. Zekavat, "Spatially correlated MIMO channel: generation via virtual channel representation," IEEE Communication Letters, vol. 10 , no. 5, pp. 332 - 334, May 2006.

[7] M. Skoglund, "Soft decoding for vector quantization over noisy channels with memory," IEEE Trans. on Inf. Theory, vol. 45, no. 4, pp. 1293-1307, May 1999.

[8] M. Skoglund and P. Hedelin, "Hadamard-based soft decoding for vector quantization over noise channels," IEEE Trans. on Inf. Theory, vol. 45, no. 2, pp. 515 - 532, Mar. 1999.

[9] L. Hanzo, et.al, OFDM and MC-CDMA for Broadband Multi-User Communications, WLANs and Broadcasting. J. Wiley Publishers, 2003. 